\title{
Gold Containers for Single Crystal Growth
}

\author{
PRODUCTION OF LEAD GERMANATE FOR INFRA-RED DETECTORS
}

\author{
G. R. Jones \\ Royal Radar Establishment, Malvern, Worcestershire, England
}

Gold crucibles, produced by electroforming, have been found to be
very satisfactory for the melt growth methods of producing single
crystals of lead germanate, a pyroelectric material used in electronic
devices such as infra-red detectors operating at room temperature.

A majority of modern electronic and optical devices makes use of the physical properties of single crystals. Such crystals may be organic, inorganic or semimetallic in nature, and they are ưsually prepared by synthetic methods. When possible the growth of a material from its own melt is the most convenient way of producing single crystals, two of the most widely used techniques for melt growth being the Bridgman-Stockbarger and the Czochralski methods. Common to these and to other methods is a container or crucible to hold the molten material. Clearly a useful crucible material must be chemically compatible with the particular substance to be contained. For example, the semiconductor gallium arsenide (GaAs) is grown from melts in silica or carbon crucibles, while the inorganic laser host oxide, yttrium aluminium garnet (YAG), is grown from melts in a metal crucible.

The binary oxide material lead germanate, $\mathrm{Pb}_{5} \mathrm{Ge}_{3} \mathrm{O}_{11}$, has for some time been under investigation at the Royal Radar Establishment for electronic applications. Crystals of lead germanate belong to a class of substances which are ferroelectric and therefore the crystals exhibit an effect known as pyroelectricity. Although this effect, which is quite literally the production of electricity or charge by heating or cooling, has been known for almost 2300 years-the Greeks knew that a crystal of tourmaline, heated in a fire and then allowed to cool, would attract particles of charcoal or ash-it is only very recently that it has assumed importance. The pyroelectric effect may be exploited in various electronic devices such as sensitive broadband infra-red detectors operating at room temperature (1).

Single crystals of lead germanate can be produced from its own melt and they have been grown using the two methods mentioned above. The usual crucible materials for oxide crystal growth are the platinum group metals $(2,3)$, of which platinum and iridium are the most widely used. However, lead germanate crystals produced from melts in platinum containers under oxidising conditions (that is in air or oxygen atmospheres) were invariably found to contain black inclusions. These inclusions, which appeared as dark coloured bands, caused strain and cracking in the crystals and so impaired their optical quality. Subsequent chemical analysis indicated that platinum was present as an impurity, possibly in the form of a platinum oxide or colloidal platinum, presumably due to the molten lead germanate interacting chemically with the surfaces of the platinum crucible. As the pyroelectric devices required large and rather perfect crystals this problem had to be solved and an alternative crucible material was sought.

Gold does not appear to be widely used for the growth of oxide materials, probably due to its relatively low melting point $\left(1063^{\circ} \mathrm{C}\right)$ in comparison to that for most refractory oxides or mixtures of oxides. The very low melting point of lead germanate $\left(740^{\circ} \mathrm{C}\right)$ and the chemical inertness of gold suggested that this metal might be a useful crucible material, while gold also offered some advantage in cost over the platinum group metals. In addition it may be produced in unusual crucible shapes by the process of electroforming.

The Bridgman-Stockbarger or normal freeze technique employs a vertical furnace with two temperature zones. The furnace is arranged so that the upper temperature zone is maintained about $50^{\circ} \mathrm{C}$ above the melting point of the material to be grown, while the lower or annealing zone is kept about $50^{\circ} \mathrm{C}$ below. Thus a temperature gradient is maintained between the upper and lower portions of the furnace, with the melting point of the material to be grown approximately midway along the gradient. The crucible containing powdered material is placed in the upper temperature region and a melt is formed. The crucible is then lowered slowly down the furnace. When the tip of the crucible passes through 
Fig. 3 In certain conditions very small inclusions of gold are found in the lead germanate single crystals. This inclusion, just below the surface of a polished section, was found to be a tiny single crystal of gold, but its method of formation and inclusion in the growing lead germanate crystal are not yet understood

that leakage would not occur during the crystal growth run. It was found that crystalline lead germanate did not adhere to the gold, and the metal could easily be peeled from the crystal boule. The crucibles were successfully used to produce crystals of lead germanate in oxidising atmospheres, with no apparent chemical reaction with the gold.

Gold crucibles have been used in the Czochralski or vertical pulling method. In this case, a seed is dipped into the molten material and by careful control of the melt temperature and the seed rotation and the rate at which the seed is lifted from the melt a single crystal can be grown (for a more complete description of the method see reference (3)). The seed is usually a crystallographically orientated rod of the material being grown. Figure 2 shows a typical pulled crystal of lead germanate grown on the hexagonal $\{0001\}$ or "c" direction. Standard Johnson Matthey Metals gold crucibles were used, these being of a straight sided, flat bottomed design generally employed with the Czochralski method. It was found that the weight of the melt (lead germanate has a density of $7.5 \mathrm{~g} / \mathrm{cm}^{3}$ ) caused a free standing gold crucible to deform. This was easily resolved, however, by placing the crucible in an alumina support or by using the gold crucible as a "liner" in a platinum crucible.

The pulled crystals were generally of good quality, but under certain conditions very small inclusions, apparently of metallic gold, having a well defined crystallographic appearance were present in the crystals. Figure 3 shows an inclusion just below the surface of a polished section of crystal. Similar inclusions have also been found in crystals of other oxide materials grown from melts contained in platinum and iridium crucibles. Apparently little is known of the nature or formation of these platelet inclusions. Careful dissolution of lead germanate in mineral acid enabled these plates to be removed and examined, and scanning electron microscope techniques revealed that the inclusions were gold while no evidence was obtained for the presence of lead or germanium (4). The inclusions were generally less than 20 microns across, with a thickness around 0.5 micron. Transmission electron microscopy indicated that the platelets were single crystals, with the cubic $\{111\}$ direction perpendicular to the plate. Exactly how these tiny single crystals of gold

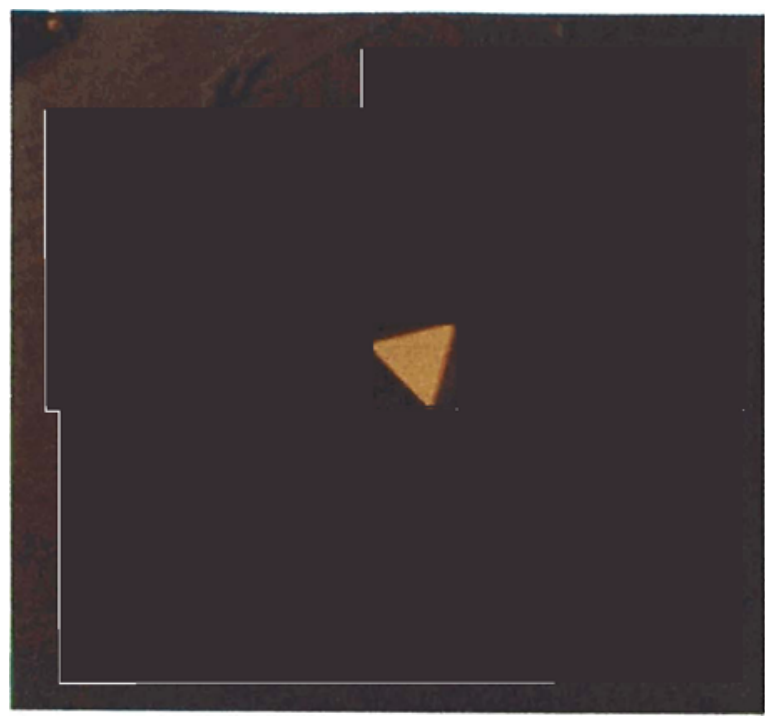

are formed and subsequently incorporated in the growing crystal is still a matter for speculation, but with iridium there is evidence that the particles may result from a volatile iridium oxide. It is difficult to see how such a situation can occur with gold, as a volatile gold oxide stable at high temperatures is not known. It is possible that gold may be removed from the crucible walls by some chemical action of the melt (or is present in the melt in colloidal form) and transported to the growing crystal interface as an unstable complex. The tendency of gold to form colloids is well known and under these conditions the charged colloid could be discharged at dislocations being formed at the growing crystal interface. The gold is hence decorating the inclusions in much the same way as some dislocation etches. These inclusions were not present in crystals of lead germanate produced by the Bridgman-Stockbarger method. Clearly a more detailed study of this phenomena is required before a complete explanation can be found.

Thus gold crucibles have been successfully employed for the growth of single crystals of the oxide ferroelectric lead germanate. It appears that other applications for gold in crystal growing may be limited by its relatively low melting point, but other low melting point materials such as lead pyrophosphate are also of interest to the electronic engineer and gold could well be found very useful in these cases also.

Figures $1-3$ are Crown Copyright, published by permission of the Director, Royal Radar Establishment.

\section{References}

1 E. H. Putley, Optic and Laser Technology, 1971, (August), 150-156

2 L. G. Van Uitert, Platinum Metals Rev., 1970, 14, $118-121$

3 B. Cockayne, Platinum Metals Rev., 1968, 12, 16-19

4 D. G. Coates, unpublished results 